\title{
ALGO SE ESTÁ MOVIENDO EN LOS HOSPITALES, ¿SERÁ QUIZÁS LA HUMANIZACIÓN CLÍNICA?
}

\author{
Dra.Antonia Pades Jiménez (*), Catalina Homar Amengual $(* *)$,
}

A. Martín-Perdiz, $\left({ }^{* * *}\right)$, E. Ponsell-Vicens (*), I. Riquelme-Agullo(***)

(*) Profesora Titular Escuela Universitaria de Enfermería y Fisioterapia.

Departamento de Enfermería y Fisioterapia

${ }^{(* *)}$ Profesora asociada de la Escuela Universitaria de Enfermería y Fisioterapia.

Departamento de Enfermería y Fisioterapia

(**) Profesora Colaboradora de la Escuela Universitaria de Enfermería y

Fisioterapia. Departamento de Enfermería y Fisioterapia

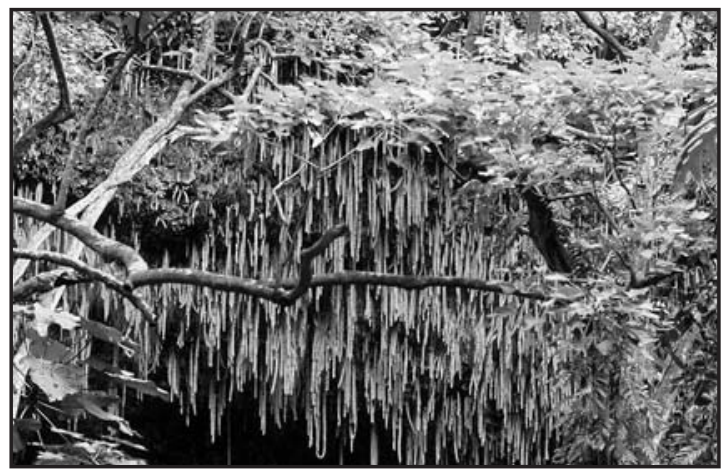

SOMETHING IS MOVING IN HOSPITALS. COULD IT BE CLINICAL HUMANISATION?

\section{SUMMARY}

I n this article results are presented after analising the students' answers in regard to perception, beliefs and attitudes on the Clinical Humanisation process in hospitals. We conclude that "something is moving", though very slowly, concerning the change in the process of humanisation of care. Students perceive that families and carers in hospitals are not well taken care of, and consider this change as essential. They believe it is necessary to acquire knowledge and comunication and social strategies towards the emotional approach to patients and their relatives.

Key words: Clinical humanisation, attitudes, beliefs, perception, psicosocial care, comunication abilities.

\section{ALGO ESTÁ OCORRENDO EM HOSPI- TAIS: TALVEZ A HUMANIZAÇÃO CLÍNICA?}

\section{RESUMO}

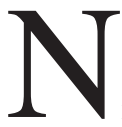

esse artigo apresentam-se resultados obtidos da análise de respostas do alunado, em relação à percepção, crenças e atitudes sobre o processo de Humanização Clínica nos hospitais. Conclui-se que "está ocorrendo algo", ainda que de maneira muito lenta, atendendo à mudança no processo de humanização dos cuidados. Os estudantes percebem que não se atende de maneira adequada à família e ao cuidador nos hospitais, e consideram imprescindível essa mudança de perspectiva. Eles acreditam que seja fundamental a aquisição de conhecimentos e estratégias comunicativas e sociais para a abordagem emocional em relação ao paciente e sua família.

Palabras-chave: Humanização clínica; atitudes; crenças; percepções; cuidados psico-sociais; habilidades comunicativas.

\section{RESUMEN}

$\square$ n este artículo se presentan los resultados tras analizar las respuestas del alumnado en relación a la percepción, creencias y actitudes sobre el proceso de Humanización Clínica en los hospitales. Se concluye que "algo se está moviendo", aunque de manera muy lenta, atendiendo al cambio en el proceso de humanización 
de los cuidados. Los estudiantes perciben que no se atiende de manera adecuada a la familia y al cuidador en los hospitales, y consideran imprescindible este cambio de perspectiva. Creen fundamental la adquisición de conocimientos y estrategias comunicativas y sociales para el abordaje emocional al paciente y la familia.

Palabras clave: Humanización clínica; actitudes; creencias; percepción; cuidados psicosociales; habilidades comunicativas

\section{INTRODUCCIÓN}

El cuidar es la base de la actividad enfermera, este cuidar posee una dimensión normativa y estipulada para definirse como tarea profesional (Feito, 2005). Los cuidados como bien interno, como lo que legitima algo como propio de una profesión, es lo que da su sentido y validez social (Feito, 2005). Sin embargo, existe otro aspecto del cuidado que consideramos tan o más importante que éste, y es el que hace referencia al cuidado como actitud. Feito define ésta como una situación de sensibilidad ante la realidad, de toma de conciencia de... El cuidado es, sin duda, una forma de responsabilidad. En contra de lo que pueda parecer, la humanización del cuidado no es un movimiento de los últimos 20 años, sino que ya se recogen artículos sobre esta materia a principios de los años 70 con trabajos como los de Kassab (1970) o de Lex (1971). A nivel docente, son muchos los trabajos que se centran en los valores que debe tener la profesión y cómo enseñarse en las aulas (Pérez et al, 2002, Oliveira et al, 2006; Barbosa, 2007), pero pocos los que ahondan en trabajos prácticos con el alumnado ya sea desde una perspectiva cualitativa o cuantitativa (Motta, 2004; Nunes, 2006; Barbosa y Silva, 2007).

Desde hace ya varios años, las docentes de la asignatura Relaciones Humanas, abordamos las variables que influyen en la intervención terapéutica, asistencial y de cuidados (Pades et al, 2004). Consideramos que es fundamental educar en la actitud del cuidado. Es una de nuestras prioridades, no sólo aportar a los alumnos conocimientos teóricos, sino contribuir a aumentar su bagaje profesional con una serie de destrezas que les permitan esta actitud de cuidar, base de unos cuidados profesionales adecuados a las demandas actuales de las persona.

En este sentido y siguiendo las directrices del Espacio Europeo de Educación Superior (EEES) que aboga por una educación centrada en el rol autónomo del alumno, durante el curso académico 2007-08, y dentro de esta asignatura se han incorporado una serie de cambios metodológicos, con el objetivo de que el alumnado desde el primer día, reflexione, partiendo de la premisa que "las personas tienen capacidad para reflexionar sobre sus propias situaciones, así como para modificarlas mediante sus propios actos"(Carr,1988) trabaje, "la buena práctica es la que permite avanzar a cotas más elevadas de desarrollo profesional" (Bardají, 2008) y desarrolle una actitud crítica y responsable, con una gestión adecuada de sus emociones. Aunque estos cambios se empezaron a introducir desde el año 1999-2000 con la aplicación de un programa de entrenamiento en habilidades sociales (EHS) diseñado y adaptado a los alumnos de tercer curso (Pades A y Ferrer VA, 2002), en el presente curso académico se han introducido nuevos aspectos metodológicos y ejercicios dentro de dicho programa. A continuación, se presentan en este artículo las reflexiones realizadas por los alumnos y se proponen una serie de estrategias de mejora.

El primer día de clase se propuso al grupo completo, una lectura crítica de un texto: "La Humanidad Clínica" (Garrido, 2003). Artículo escrito por un periodista conocido a nivel local, autor también del libro: "Te lo contaré en un viaje", donde narra su experiencia como familiar y acompañante de su hija adolescente enferma hasta su muerte. A los alumnos de tercer curso tras la lectura del artículo se les propuso el ejercicio de contestar de manera abierta y tras la reflexión grupal a una serie de preguntas, con el objetivo de explorar cuál era la percepción del alumnado ante el proceso de humanización en el hospital, y analizar las actitudes y/u opiniones en relación a los cuidados prestados a la familia y/o cuidador principal.

Nuestro objetivo como docentes era entonces, explorar y analizar sus percepciones, creencias y actitudes sobre el tema, para luego insistir a nivel docente en la adquisición de nuevos conocimientos y estrategias comunicativas y de interacción social. 
El segundo objetivo, lo teníamos garantizado con el aporte de los conocimientos a nivel teórico, pero la primera parte de analizar percepciones, creencias y actitudes $\mathrm{y}$, si era preciso intentar cambiarlas era más compleja. CAMBIAR ACTITUDES, todos nosotros lo sabemos, es difícil, cuesta y sobre todo si éstas ya están adquiridas y reflejan un aprendizaje social y por observación del entorno que nos rodea (Bandura, 1986). Las propias experiencias negativas con el sistema sanitario, y con los encuentros con diferentes modelos (profesionales de la salud) que interactúan en él, nos condicionan e influyen en nuestras percepciones, actitudes, emociones y conducta (Borrell, 2003).

\section{MATERIAL Y MÉTODO}

Estudio descriptivo transversal en una muestra de 133 alumnos/as de tercer curso de enfermería de la Escuela Universitaria de Enfermería de la Universitat de les Illes Balears (UIB), durante el curso académico 2007-2008.

Tras la lectura de un texto escrito que era de corta extensión, que suponía una lectura individual de aproximadamente 4-5 minutos, se formaron grupos de 3-4 personas que reflexionaron sobre una serie de cuestiones que planteamos. Tras la clase, los alumnos debían seguir trabajando el tema, ya que les habíamos propuesto que este trabajo grupal reflexivo, tuviese una ponderación en la nota final de la asignatura. Por lo tanto, nos debían entregar sus conclusiones grupales por escrito. La propuesta exacta era que a lo largo del curso académico se realizasen trabajos de reflexión a nivel grupal de este tipo, que atendían al temario teórico que se estaba impartiendo, y que luego las conclusiones se entregarían por escrito a las profesoras para su corrección y posterior feedback. A estos trabajos se les denominó "Entregables". Este trabajo pues, suponía el primer entregable del curso. Podría servir también de ejercicio de "Calentamiento" tal y como se denominan a los ejercicios para romper el hielo (Caballo, 1986). En los procesos de inicio de la interacción social. Los alumnos aún no se habían adentrado por la asignatura teórica de Relaciones Humanas.

Para ello, las preguntas que se les formularon fueron las siguientes:
- Creéis que ALGO SE ESTÁ MOVIENDO en el hospital, refiriéndose al cambio o al movimiento humanista en la práctica asistencial, tal y como el texto apunta.

- Cómo se ve a la familia en los hospitales.

- Creéis que se cuida a los cuidadores, acompañantes.

- La valoración emocional del paciente, cliente y familia ¿se está haciendo? Vosotros como alumnos de tercer curso, ¿cómo se os ocurriría abordar este tema? Empieza evaluando... ¿qué preguntarías para conocer estos aspectos emocionales?¿Qué aspectos de la comunicación no verbal observarías y por qué? ¿cómo lo harías tú......?.

- Humanizar, es algo más que una técnica ¿Por qué?

- Qué entiendes por empatía. Defínela y describe los parámetros (verbales y no verbales) que la componen.

- Cuando el autor afirma que "sí que existe un código universal de humanidad" qué creéis que está diciendo.

- Para finalizar, qué cualidades creéis que debe tener una enfermera/enfermero para cuidar.

\section{RESULTADOS}

Los resultados más relevantes se presentan a continuación:

La mayoría de los alumnos, (98\%) perciben que "sí, algo se está moviendo pero de manera muy lenta". Textualmente comentan: "Tenemos que tratar a personas, se va dejando atrás la fórmula: paciente de la 324"....Otro alumno comenta, "Sí algo se está moviendo, hemos dejado de ver una herida para ver a una persona". "Se mueve pero de forma muy pequeña, al igual que cuando tiramos una piedra al lago, se van haciendo círculos uno más grande que el otro y a consecuencia del más pequeño. Si se cuenta con la humanización y se tiene la mentalidad que hay que cambiar esos cuidados tan técnicos es porque la piedra ya se ha tirado y poco a poco los círculos se harán más grandes"

- El 76\% perciben que aún no se ve a la familia como se tiene que ver, "como una persona o personas que necesita también cuida- 
dos". Se les ve como una molestia "más personas con las quien batallar", "como un estorbo" La visión generalizada es la de personas incordiantes y molestas. "Como un obstáculo". Se les califica como "pesados." o "listillos". Los alumnos dicen, en los hospitales, se observa una familia esquivada por parte del profesional, además de una familia desgastada tras dormir día a día en una incómoda butaca. Para la persona ingresada, una de las cosas más importantes, por no decir la más importante, es el apoyo de sus personas cercanas, ya sean familiares, pareja, amistades....y es en los que realmente se apoyan. Es como si a un anciano que usa bastón para caminar y que sin él le cuesta moverse, le quitásemos ese bastón, al final el anciano acabará cayéndose solo.

El resto de alumnos apunta a que sí que se cuida a los familiares, que se ha avanzado mucho, que se percibe un notable cambio, que la familia no es un concepto cerrado, cada hospital, cada unidad y cada profesional tiene su propia concepción de FAMILIA, y ésta puede distar mucho de unos a otros.... La mayoría concluyen que necesitamos una educación paciente-familia-personal sanitario en la que todos nos ayudemos y no nos perjudiquemos, además tenemos que ver al paciente y a la familia como un conjunto inseparable.

- A la pregunta Creéis que se cuida al cuidador/acompañante: la mayoría apunta que no (88\%) La mayoría de veces nos centramos en los cuidados físicos del paciente, nadie les pregunta a los cuidadores cómo se sienten ellos, y si necesitan alguna cosa. Identifican que se les atiende mal. Es una tarea que requiere mucho tiempo y paciencia. En los hospitales hay poco personal para la elevada carga de trabajo. Existe una notable diferencia entre el hospital y el ámbito comunitario. Otro alumno comenta que "para mí es la gran asignatura pendiente, ya que en ocasiones la carga física y emocional que recae sobre el cuidar es mayor que la que pueda sufrir el propio paciente".
La valoración emocional del paciente y familia ¿se está haciendo?

- El 38\% apunta que sí. "Que la valoración emocional del paciente va subiendo peldaños y cada vez la tenemos más en cuenta ya que el proceso de humanización nos obliga a estar más pendientes de este comportamiento emocional" Otros alumnos dicen (56\%) que sí, pero en atención primaria no en el hospital. Otros dicen que sí que se hace, pero se hace mal. "Algunas enfermeras hablan con los pacientes, pero lo hacen durante muy poco tiempo, en momentos no adecuados para hablar de sentimientos (como pueden ser la higiene, la medicación...), y después de saber cuáles son esas angustias o pensamientos del enfermo no hacen nada para mejorarlas"... "Se ven indicios de empezar a valorar tanto el estado físico como emocional"

- Cuándo se les pide cómo lo harías tú... Se formulan preguntas de manera adecuada, sin embargo confunden los parámetros a observar y las emociones que deben explorar.

- Humanizar, es algo más que una técnica ¿Por qué?

Los alumnos están de acuerdo con esta premisa, y dicen.... " Humanizar es mostrarse próximo, sereno, comprensivo". "Humanizar es algo nuestro, propio de los seres humanos. Humanizar se trata de comprender a la persona, es algo innato, no se aprende.... Es hacer humano o familiar a algo a alguien. Humanizar tu trabajo es hacerlo sabiendo que ofreces toda tu calidez como persona y de la misma forma que te gustaría recibirlo".

- Qué entiendes por empatía. Defínela y describe los parámetros (verbales y no verbales) que la componen. Apuntan entre otras..."Ponerse en el lugar del otro y comprender, ver a la persona desde su propia perspectiva, capacidad para comprender los pensamientos y las emociones del paciente". En relación a los parámetros, apuntan: escucha activa, mirada, comprensión, tacto, asentir con la cabeza, sonrisa adecuada no falsa..., coger la mano, tocar a la persona, e incluso abrazar. ... 


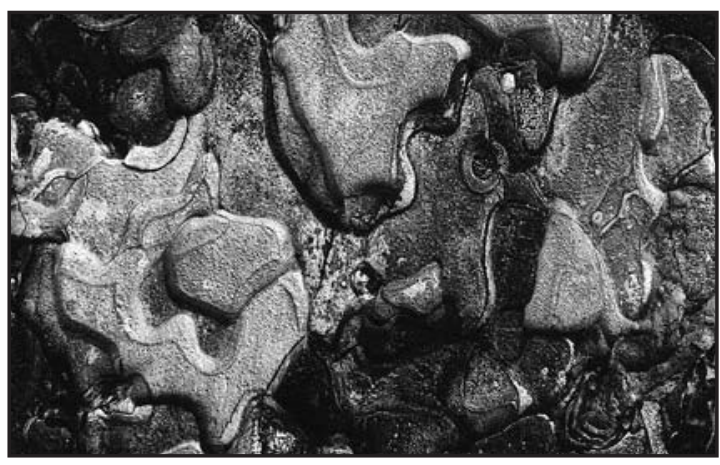

- Cuando el autor afirma que "sí que existe un código universal de humanidad" qué creéis que está diciendo. Ellos identifican como: "creo que quiere decir: dignidad, libertad de elegir, sentido de haber cumplido con la vida, afecto sin condiciones". "Valorar a la persona de forma integral, forma más cercana, atenta y dándole la posibilidad de dar su opinión y respetarla. Humanizar no es algo mecánico". "Se consigue implicándose cada uno, sacando lo mejor de uno mismo". "Creo que existe un código con el que todos los humanos somos capaces de entendernos, incluso a veces sin hablar: no hace falta ser un profesional muy cualificado en técnicas muy complicadas, para hacer sentir a alguien que estás a su lado, y que tiene todo tu apoyo y comprensión".

- Para finalizar, analizar qué cualidades creéis que debe tener una enfermera/enfermero para cuidar. Las cualidades que creen que son necesarias para que una enfermera/o pueda cuidar son: Saber escuchar, empatizar, amabilidad, respeto, sensibilidad, comprensiva, tolerancia, saber trabajar en equipo, segura de sí misma, y preocupación por la problemática de la familia....

\section{DISCUSIÓN}

Como docentes, interpretamos que el alumnado realiza un juicio de valor negativo hacia los profesionales de la salud del entorno hospitalario, y que generalizan. Esto particularmente nos preocupa teniendo en cuenta que esta actitud o percepción tan negativa, no solo condiciona sus conductas sino también sus emociones. Nuestro propósito será intentar qué sepan adquirir una actitud crítica, pero constructiva, que sepan identificar los elementos que pueden influir en el proceso de Humanización, y sobre todo, que sean capaces también de ser autocríticos con ellos mismos e intentar despertar el interés y la motivación hacia el proceso de humanización. La humanización no es algo que suceda de manera espontánea sino que debe ser fomentada, aprendida y practicada (Escuredo, 2003). Como reflexiona Santos Hernández (2003) “ Sólo un ser humano es capaz de reconocer la dignidad de otro y respetar sus derechos, asumiendo en consecuencia el deber ético de asistirlo, cuidarlo y consolarlo y acompañarlo en su fragilidad ".Sin embargo, para conseguir esta interacción este" Abrir ( paciente) Donar (enfermera - profesional)"Es imprescindible que se conjuguen: vocación, competencia profesional y virtudes morales (Los alumnos creen necesaria una formación rigurosa sobre el tema, sin embargo, nosotras pensamos que la formación y conocimientos teóricos no bastan, se necesita también QUERER, se necesitan actitudes positivas y estar motivados. El componente observacional tiene un valor importante e intrínseco en el aprendizaje (Bandura, 1986). HAGO LO QUE VEO. Los modelos representativos, incluso durante las prácticas clínicas, son esenciales. El ver cómo lo hacen las enfermeras y médicos es fundamental.... Pero también observar a sus propias profesoras. Debemos ser y dar ejemplo. Siguiendo la propuesta de Escuredo (2003) además de lo que se enseña de manera formal (consciente) existe otro elemento a tener en cuenta en el análisis del aprendizaje de los valores profesionales: el denominado currículo oculto (Espindler, 1993). Que incluye lo que transmitimos los profesionales durante las prácticas clínicas y los profesores en el aula a los alumnos mediante nuestras propias actitudes, la priorización que hacemos de nuestros temas, la selección de lecturas, etc. Los profesionales deben ser muy conscientes de lo que transmiten al futuro profesional, así pues será otro elemento a tener en cuenta en la humanización de los cuidados.

\section{CONCLUSIONES}

- Los alumnos argumentan: Sí que algo se está moviendo en el hospital, en relación a la 
humanización, pero de manera muy lenta. Dicen que la gente (se refieren a los profesionales de la salud) está más sensibilizada, pero tienen pocos recursos para poner en práctica el proceso... La organización no lo favorece."

- NO SE CUIDA en el medio HOSPITALARIO a los que CUIDAN. Consideran que se ve a la familia como "un estorbo", y que cuando no quitan el trabajo del profesional, dicen textualmente, "molestan..."

- Sin embargo, consideran que es necesario y útil que la familia se tome en cuenta como PILAR fundamental en el proceso de cuidados y recuperación del paciente y como fuente de apoyo emocional indiscutible.

- Los alumnos identifican como MUY NECESARIOS y también útil la formación en áreas de comunicación terapéutica, valoración y entrevista clínica y psicología.

- Identifican cualidades humanas como relevantes y necesarias. Las cualidades que creen que son necesarias para que una enfermera/o pueda cuidar son: Saber escuchar, empatizar, amabilidad, respeto, sensibilidad, comprensiva, tolerancia, saber trabajar en equipo, segura de sí misma, y preocupación por la problemática de la familia.... Estos resultados coinciden con los hallazgos de (Pérez A et al., 2002) también en una muestra de alumnos de enfermería.

Para finalizar creemos que es necesario reflexionar, discutir, insistir y formar en valores humanistas, para que nuestro alumnado reflexione, sean críticos, autocríticos y autónomos como personas y como profesionales. El humanismo implica una actitud frente a la vida. Sería interesante seguir investigando, y replicar este estudio con alumnado de enfermería de otras Escuelas.

\section{BIBLIOGRAFÍA}

- Bandura, A. (1987) Teoría del Aprendizaje Social. EspasaCalpe, Madrid

- Barbosa IA, Silva MJ. (2007). Humanization in nursing care: acting with respect in a school hospital. Rev Bras Enferm. 2007 Sep-Oct;60(5):546-51.

- Bardaji, T. (2008). El diario reflexivo como herramienta de autoaprendizaje en la formación de enfermería. Nursing 26, 52-55.
- Borrell, F.(2004) ENTREVISTA CLINICA. Manual de estrategias prácticas. semFYC, Barcelona.

- Caballo, V.E.(1993) Manual de evaluación y entrenamiento de las habilidades sociales. Siglo Veintiuno de España Editores, Madrid.

- Carr W, Femmis S.(1988) Teoría crítica de la enseñanza. Martínez Roca, Barcelona.

- Escudero, B. (2003) Humanismo y tecnología en los cuidados de enfermería desde la perspectiva docente. Enf. Clínica 13, 164-70.

- Espindler, GD. (1993) La transmisión de la cultura. Trotta, Madrid

- Fargues i Garcia I.(2002) El diario reflexivo y el autoaprendizaje tutorizado. Metas Enferm. 45:14-8.

- Feito L. (2005) Los cuidados en la ética del siglo XXI. Enf Clínica 15(3), 167-174

- Garrido C (2003) La humanidad clínica. Tempos Vitalis. Revista Internacional para el Cuidado del Paciente Crítico 2(3):49-50.

- Kassab P. (1970). Efficiency, productivity and humanization of the medical assistance system. AMB Rev Assoc Med Bras 16(11), 381-382.

- Lex A. (1971). Humanization of hospitals: respect for the human personality. Rev Hosp Clin Fac Med Sao Paulo 26(2), 83-86.

- Motta MG. (2004). Humanized care in the teaching of nursing. Rev Bras Enferm. 57(6), 758-760.

- Nunes DM, Wegner W, Bottan G. (2006). How nursing professors insert the theoretical content on human care into the development of their discipline. Rev Gaucha Enferm 27(4), 539-547.

- Oliveira BR, Collet N, Viera CS. (2006). Humanization in healt care. Rev Lat Am Enfermagem 14(2), 277-284.

- Pades A, Ferrer VA. (2002) Cómo mejorar las habilidades sociales. Ejercicios prácticos para profesionales de enfermería. Grupo Editorial Universitario, Granada

- Pades A, Amorós SM, Homar C. (2004). Actitudes y cualidades esperadas en los profesionales enfermeros. Metas de Enfermería 7(5), 54-58.

- Pérez et al. (2002) Salud y Cuidados. 3. http://www.saludycuidados.net/numero3/perfilvalores/perfilvalores.htm consultado $[06 / 05 / 08$

- Santos, A. M. (2003) Humanización de la Atención sanitaria: reto y perspectivas. Cuadernos Bioética 14, 39-60.

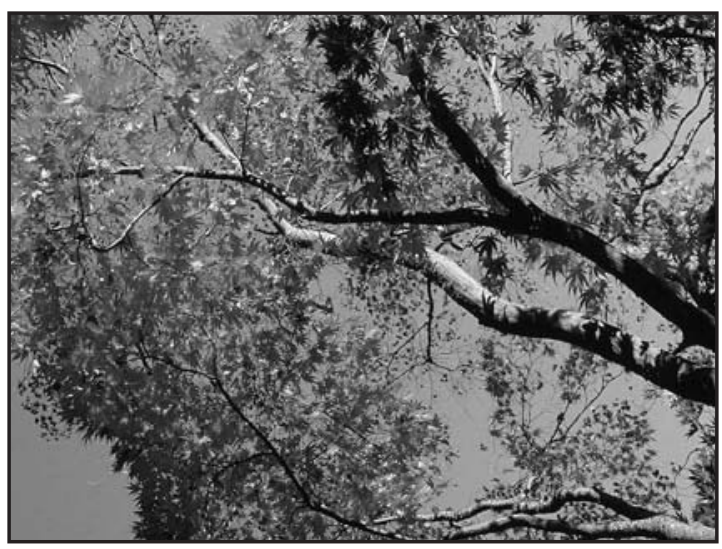

